University of Nebraska - Lincoln

DigitalCommons@University of Nebraska - Lincoln

Faculty Publications: Materials Research

Science and Engineering Center

Materials Research Science and Engineering

Center

$12-22-2006$

\title{
Evidence of Long-Wavelength Collective Excitations in Magnetic Superlattices
}

\author{
Nikolay I. Polushkin \\ University of Nebraska - Lincoln \\ Steven A. Michalski \\ University of Nebraska - Lincoln, smichalski2@unl.edu \\ Lanping Yue \\ University of Nebraska - Lincoln, lyue2@unl.edu \\ Roger D. Kirby \\ University of Nebraska - Lincoln, rkirby1@unl.edu
}

Follow this and additional works at: https://digitalcommons.unl.edu/mrsecfacpubs

Part of the Materials Science and Engineering Commons

Polushkin, Nikolay I.; Michalski, Steven A.; Yue, Lanping; and Kirby, Roger D., "Evidence of LongWavelength Collective Excitations in Magnetic Superlattices" (2006). Faculty Publications: Materials Research Science and Engineering Center. 63.

https://digitalcommons.unl.edu/mrsecfacpubs/63

This Article is brought to you for free and open access by the Materials Research Science and Engineering Center at DigitalCommons@University of Nebraska - Lincoln. It has been accepted for inclusion in Faculty Publications: Materials Research Science and Engineering Center by an authorized administrator of DigitalCommons@University of Nebraska - Lincoln. 


\title{
Evidence of Long-Wavelength Collective Excitations in Magnetic Superlattices
}

\author{
Nikolay I. Polushkin, ${ }^{1,2}$ Steven A. Michalski, ${ }^{1}$ Lanping Yue,, 3 and Roger D. Kirby ${ }^{1}$ \\ ${ }^{1}$ Department of Physics and Astronomy, University of Nebraska, Lincoln, Nebraska 68588-0111, USA \\ ${ }^{2}$ Institute for Physics of Microstructures, Russian Academy of Sciences, 603950 GSP-105, Nizhniy Novgorod, Russia \\ ${ }^{3}$ Nebraska Center for Materials and Nanoscience, University of Nebraska, Lincoln, Nebraska 68588-0111, USA
}

(Received 24 July 2006; published 18 December 2006)

\begin{abstract}
We report on a mechanism of dynamic dipolar coupling in magnetic superlattices via long-wavelength nonevanescent fields. In the spin excitation spectra of our heterophase stripe structures, such interactions mediate a singlet $\leftrightarrow$ doublet crossover in the frequency regime driven by the orientation of an external static field. This crossover is a new feature observed in collective behavior of superlattices, though there is some analogy of this phenomenon with birefringence taking place in optical superlattices. We envision applying the collective effects described here in microwave photonic devices.
\end{abstract}

PACS numbers: 71.45.- d, 72.15.Nj, 73.20.Mf, 75.30.Ds

The spectrum of collective excitations in solids with spatial modulation of their physical properties (superlattice structures) strongly depends on the product of the wave number $Q$ and modulation period $D$ [1-4]. In magnetic structures, a well-established mechanism of (super)collective excitations is the interlayer interactions via evanescent dipolar fields [5-7]. These fields having $Q \sim 1 / D$ originate from the surface modes propagating along the layers in vertical structures (deposited multilayers). In the longwavelength limit $(Q D \ll 1)$, the fields tend to reduce to zero [8], so the spectrum reduces to a simple summation of independent contributions of each constituent in a superlattice. Different behavior could be expected in lateral structures (patterned films), in which the modes are able to propagate across the interfaces providing common components of the long-wavelength fields $[9,10]$. However, the wave numbers of the modes excited in real finite systems are conventionally limited to $Q \sim 1 / D$ because of spin pinning at the interfaces [11,12]. Basically, any experimental observation of long-wavelength collective excitations would be of general interest because the long-wavelength approximation is equivalent to substituting a superlattice by a homogeneous anisotropic medium $[13,14]$. This anisotropy, for instance, in optical superlattices gives rise to their useful properties (e.g., birefringence) [14].

Here we present a real magnetic system that falls out of the conventional behavior and demonstrates the features of collective excitations associated with the long-wavelength limit (LWL). Our samples are lateral heterostructures patterned directly by laser beams in Fe-based alloyed films [15] and having one-dimensional periodic modulation of the static magnetization $M$. In the spin excitation spectra of such systems, we find a crossover from the two-frequency (doublet) to single-frequency (singlet) peak, as the orientation of an external magnetic field $\boldsymbol{H}$ is changed with respect to the direction of periodicity. Qualitatively, we explain the observed crossover by the effects of the longwavelength $(Q D \ll 1)$ nonevanescent fields [that is, these fields in bulk are given by $\boldsymbol{h}=-4 \pi(\boldsymbol{n} \cdot \boldsymbol{m}) \boldsymbol{n}$, where $\boldsymbol{m}$ is the dynamic magnetization and $\boldsymbol{n}$ the unit vector along the direction of propagation]. With a reorientation of $\boldsymbol{H}$, dynamic field components alter to obey the usual electromagnetic boundary conditions at the interfaces between the constituents. This ability results in the drastic changes in the excitation spectrum we report here. To quantitatively explain the observed behavior, we have calculated the eigenfrequencies in the LWL approximation $[9,10]$ of a lattice of two subsystems of stripes having different magnetizations. In other words, we ignore the effects of spin pinning at the interfaces of our systems. Surprisingly, we find both qualitative and quantitative agreement of the experimental data with results of our calculations. The obtained results are stimulating for both further fundamental research of spin dynamics in magnetic superlattices and possible applications in microwave magnetic devices [16].

Our samples are prepared in two steps. The first step was pulsed laser deposition of small $(\sim 1 \AA)$ portions of magnetic $(\mathrm{Fe})$ and nonmagnetic $(\mathrm{V})$ species alternatively onto $\mathrm{Si}$ substrates. The total film thickness was varied in the
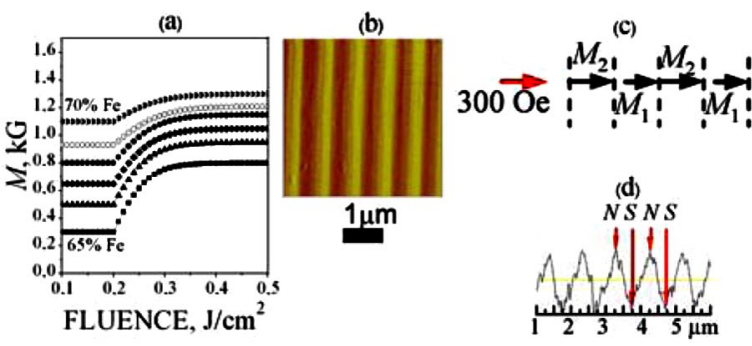

FIG. 1 (color online). Initial sample characterization. (a) Laser-induced enhancement of the magnetization $M$ in Fe$\mathrm{V}$ layers at different $\mathrm{Fe}$ content in the range of $65 \%-70 \%$. (b) MFM image of a $0.9-\mu \mathrm{m}$-period stripe lattice patterned directly by two interfering laser beams in a 12-nm-thick Fe-V layer. (c) Schematic view of distribution of the magnetizations in a 300 Oe field applied in situ. (d) Cross section of the MFM response of the patterns, which is indicative of the ratio of stripe widths, as distances between $\mathrm{N}$ and $\mathrm{S}$ poles. 
range from a few tens to a few hundred nanometers. Shortpulse laser annealing of such alloyed films initiates a phase separation in them on the scale of a few nanometers and growth of Fe-enriched clusters [17]. These rearrangements, in turn, cause an enhancement of the overall magnetization $M$ measured at room temperatures. The magnetization in annealed samples depends on both the initial magnetization (Fe content) and the intensity of laser annealing. As shown in Fig. 1(a), laser-induced increase in the magnetization starts at the threshold fluence of $\sim 0.2 \mathrm{~J} / \mathrm{cm}^{2}$ for different $\mathrm{Fe}$ contents. Films with compositions of $65 \%-$ $70 \% \mathrm{Fe}$ are the most interesting for patterning. Above $70 \%$ of Fe the samples do not exhibit pronounced modification in the magnetic properties, whereas below $65 \%$ of $\mathrm{Fe}$ the as-deposited samples are not ferromagnetic (at least, at room temperatures).

The second step was direct patterning of the asdeposited films over areas of $\sim 1 \mathrm{MM}^{2}$ with a single laser shot of nanosecond duration by two interfering beams of approximately equal intensity with a radiation wavelength of $532 \mathrm{~nm}$ (the second harmonic of a Nd:YAG laser). At fluences close to the threshold, the film magnetization was changed mostly in interference maxima. In Fig. 1(b) we show a typical magnetic force microscopy (MFM) image of a $0.9-\mathrm{mm}$-period lattice of stripes patterned in a 12-nmthick $\mathrm{Fe}(65 \%)-\mathrm{V}$ sample. To take the image with North (N) and South (S) magnetic poles pronounced at the interfaces, the magnetization was saturated by $300-O e$ field applied in situ along the direction transverse to the interfaces [Fig. 1(c)]. As the cross-section analysis of the MFM imaging reveals, the ratio of the stripe width to the lattice period is about 0.5 [Fig. 1(d)].

To measure the spin excitation spectra, we employ a pump-probe technique with direct optical excitation of spin precession [18], which is based on a MIRA 900 femtosecond laser system (a pulse duration is $150 \mathrm{fs}$, radiation wavelength is $800 \mathrm{~nm}$, and power output is $0.8 \mathrm{~W}$ ). In these

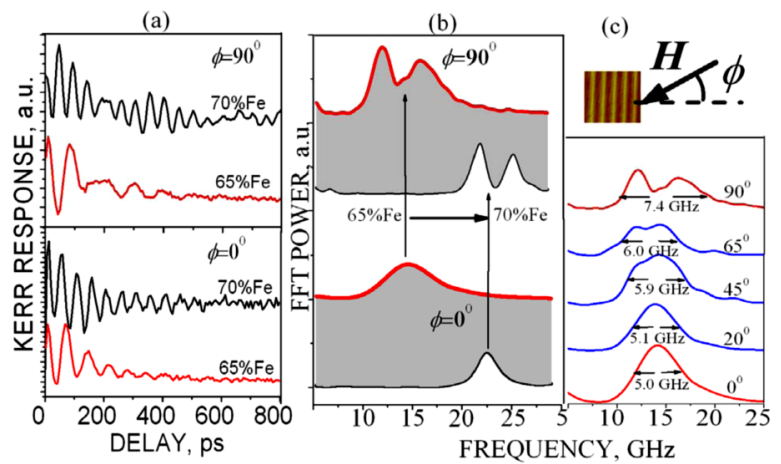

FIG. 2 (color online). Crossover in the excitation spectra. (a) Magneto-optical Kerr response of patterned $\mathrm{Fe}-\mathrm{V}$ films, at the two edges of Fe compositions (70\% and $65 \%$ ), as a function of time delay in the two particular configurations of $\boldsymbol{H}: \phi=90^{\circ}$ and $\phi=0^{\circ}$. (b) The corresponding FFT spectra at $\phi=0^{\circ}$ (lower curves) and $\phi=90^{\circ}$ (upper curves). (c) FFT spectra taken at different $\phi$ for a sample with $\mathrm{Fe}$ content of $65 \%$. experiments, the pump and probe beams were focused to spots with diameters of $10-20 \mu \mathrm{m}$ and then scrupulously overlapped. The diameter of pump spot was optimized so that spin precession could effectively be excited and, on the other hand, the LWL could still be achieved experimentally by retaining the spot size much larger than the period of our structures $D$. The dynamic magnetization was measured by detection of transient magneto-optical Kerr response at different orientation angles $\phi$ of $\boldsymbol{H}$ with respect to the stripes. The field was slightly tilted out of plane to induce a canted orientation of the magnetization and thus to increase the signal-to-noise ratio [18].

Figure 2(a) shows the Kerr response measured as a function of pump-probe delay at $H=3.0 \mathrm{kG}$ for the lattices at the two edges of the range of Fe concentrations (70\% and $65 \%$ ). The spectra are shown for two particular geometries, parallel and transverse orientations of $\boldsymbol{H}$ with respect to the stripes. We mark a drastic change in the behavior of precession decay with a reorientation of $\boldsymbol{H}$. Indeed, in the parallel configuration $\left(\phi=90^{\circ}\right)$ one observes beats in the oscillations, whereas in the other geometry $\left(\phi=0^{\circ}\right)$, the decay occurs in monotonous manner. The modal structure of the motion is immediately apparent from the time domain. In Figs. 2(b) and 2(c) we show the fast Fourier transformation (FFT) of the spectra corresponding to the parallel and transverse configurations (upper and lower curves, respectively) (b), as well as to the spectra taken at other angles $\phi$ (c). As seen, the frequency spectra indicate a singlet state at $\phi=0^{\circ}$. As the orientation of $\boldsymbol{H}$ is changed to the parallel configuration $\left(\phi=90^{\circ}\right)$, we observe a gradual broadening of the single-frequency peak with a subsequent crossover to the doublet state with a maximum splitting of $\Delta f_{\exp }=3.8 \pm$ $0.3 \mathrm{GHz}$ at $\phi=90^{\circ}$. These features - the broadening and

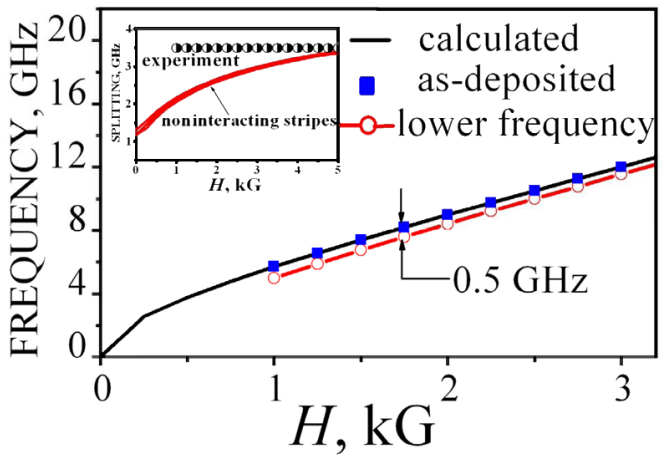

FIG. 3 (color online). Two-frequency regime: modified frequencies. Comparison of measured frequencies of the lower peak with those in the as-deposited sample. The frequencies are given as functions of $H$. The solid curve is a fit to the measured frequencies in the as-deposited sample with $M=$ $0.25 \mathrm{kG}(65 \% \mathrm{Fe})$ and $\gamma=2.8 \mathrm{GHz} / \mathrm{kG}$ according to $\omega=$ $\gamma H(H+4 \pi M)^{1 / 2}$. Inset: Measured deviation of the difference between upper and lower frequency in the double peak from that difference for noninteracting stripes calculated from Eq. (5) in Ref. [12]. 
splitting of the frequency peak - are of potential interest to use such heterostructures in microwave magnetic filters [16] whose bandpass could be varied by the reorientation of $\boldsymbol{H}$.

Another experimental observation is that the measured frequencies in the two-frequency regime $\left(\phi=90^{\circ}\right)$ differ from those which would be in a system of noninteracting stripes. We find that the lower frequency is lower $(\approx 0.5 \mathrm{GHz})$ than that in the as-deposited sample (Fig. 3). Here we also show the frequencies in a homogeneous medium, which are calculated as $\omega=\gamma[H(H+$ $4 \pi M)]^{1 / 2}$ and fitted to the measured frequencies in the as-deposited sample (solid curve). Laser annealing, however, causes only an enhancement of the magnetization [Fig. 1(a)] and thus an increase of the precession frequency. Therefore, interstripe interactions should be taken in account. Furthermore, we find a difference between the measured spitting in the double-frequency peak and the calculated difference in the frequencies in a heterosystem of noninteracting stripes. These quantities are shown as functions of $H$ in inset of Fig. 3. As seen, the measured splitting is practically constant for a broad range of $H$. In contrast, the difference calculated for noninteracting stripes, taking in account spin pinning at their edges [Eq. (5) in Ref. [12]] (solid curve in inset of Fig. 3), strongly decreases with lowering $H$. The aspect ratio of stripes (thickness to width) taken for calculation is $0.020 \pm$ 0.005 , which corresponds to the real parameter. The error we evaluate for the aspect ratio is shown by appropriate thickening of the curve.

To understand the obtained experimental results, we develop a theoretical model upon the basis of earlier treatments of magnetic superlattices within the LWL approximation $[9,10]$. As a model system, we consider a thin layer of thickness $t$, which consists of alternating stripes of two different magnetic materials with widths $w_{j}$, saturation magnetizations $M_{j}$, and gyromagnetic ratios $\gamma_{j}$, where $j=$ 1,2 . The length of stripes is assumed to be infinite compared to the two other dimensions. The stripes are incorporated into dense arrays but without direct contact between adjacent stripes (i.e., no exchange coupling between the stripes) to form a superlattice with a period $D=w_{1}+w_{2}$. The lattice lies and is rotated in the $x z$ plane, and $\boldsymbol{H}$ is oriented along the $z$ axis. As $t \ll D$ under our experimental conditions, one can neglect the in-plane static demagnetizing factors and thus can assume that $\boldsymbol{M}_{1,2}$ is oriented along $\boldsymbol{H}$. While neglecting all exchange terms, the effective field in each kind of stripes consists of only two terms: the Zeeman field $\boldsymbol{H}$ and dynamic dipolar field $\boldsymbol{h}_{j}$. Since $\boldsymbol{m}_{j}$ alter in space because of the nonuniformity of pumping over the excited location, generated $\boldsymbol{h}_{j}$ have the nonzero long-wavelength components in the lattice plane. In this case, the permeability tensor for each of two constituents takes the form given, for instance, in Refs. [9,10]. In our rigorous analysis we ignore the pinning effects at the interfaces and assume that the quantities $\boldsymbol{m}_{j}$ and $\boldsymbol{h}_{j}$ are constants within the first and second media over a whole period of the superlattice. Since the tangential component of dipolar field and the normal component of magnetic induction are continuous across interfaces, the appropriate field components are everywhere equal to their average values. Then, the other field components can be expressed via these common fields and averaged over the period. This standard procedure gives the effective-medium permeability tensor [9], whose components connect the averaged values of the fields. The nonzero component of the tensor, which is very essential in our considerations, is given by

$$
\mu_{y y}^{\mathrm{eff}}= \begin{cases}r \chi_{1}+(1-r) \chi_{2}, & \phi=0, \\ B^{2} / A+C, & \phi=\pi / 2,\end{cases}
$$

where $A=r / \chi_{1}+(1-r) / \chi_{2}, B=r \nu_{1} / \chi_{1}+(1-r) \nu_{2} / \chi_{2}$, $C=r \eta_{1}+(1-r) \eta_{2}, \quad \eta_{j}=\left(\chi_{j}^{2}-\nu_{j}^{2}\right) / \chi_{j}, \quad \chi_{j}=$ $1+\Omega_{H(j)} /\left(\Omega_{H(j)}^{2}-\Omega_{j}^{2}\right), \nu_{j}=1+\Omega_{(j)} /\left(\Omega_{H(j)}^{2}-\Omega_{j}^{2}\right)$, $\Omega_{H(j)}=H / 4 \pi M_{j}, \Omega_{j}=\omega /\left(4 \pi \gamma_{j} M_{j}\right)$, and $r=w_{1} / D$.

It is crucial to understand that, in the transverse configuration $(\phi=0)$, the effective medium is isotropic $\left(\mu_{x x}^{\text {eff }}=\right.$ $\mu_{y y}^{\text {eff }}$ ) and all the effective-medium tensor components result from simple averaging over the superlattice period. In this sense, the transverse configuration is analogous to the ordinary beam propagating in a birefringent medium. The electromagnetic boundary conditions applied to such a geometry provide a unified dipolar field in the system and thus the same precession frequency for the both constituents. However, in the parallel configuration, the effective medium becomes anisotropic $\left(\mu_{x x}^{\text {eff }} \neq \mu_{y y}^{\text {eff }}\right)$ (extraordinary beam) and a difference $4 \pi\left(m_{x(2)}-m_{x(1)}\right)$ arises between the dipole-field components transverse to the interfaces. To understand the difference in the modal structure between these two geometries, we look for plane-
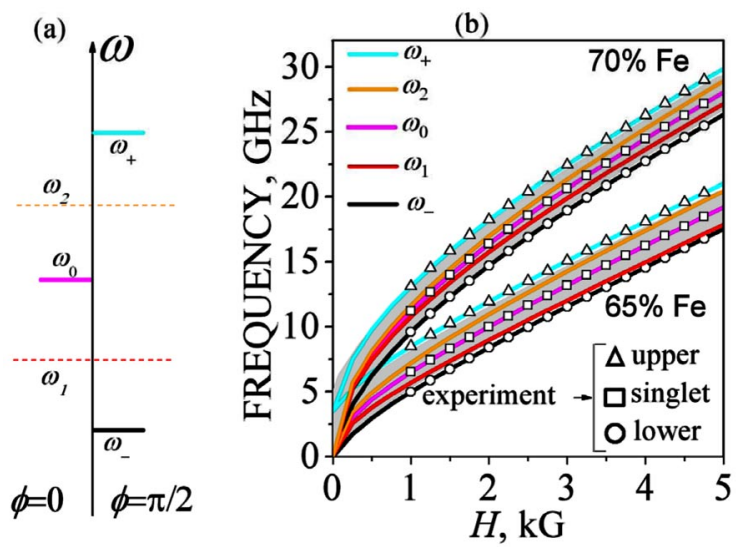

FIG. 4 (color online). General picture of the frequency behavior. (a) Schematic diagram of the calculated frequencies in a lateral superlattice heterostructure with two different magnetizations. (b) $\omega_{-}, \omega_{0}$, and $\omega_{+}$as functions of $H$ at $M_{1}=0.25 \mathrm{kG}$ and $M_{2}=0.45 \mathrm{kG}$ (lower band) as well as $M_{1}=1.1 \mathrm{kG}$ and $M_{2}=1.3 \mathrm{kG}$ (upper band). These frequencies correspond to the measured frequencies (shown by symbols) in patterned $\mathrm{Fe}-\mathrm{V}$ films with $\mathrm{Fe}$ content of $65 \%$ and $70 \%$. 
wave solutions [19] of the Walker equation for the effective medium. Substitution of these solutions into the Walker equation while taking into account the thin-film geometry leads to the dispersion relations for the guided and surface modes, which are identical to those derived in Ref. [10]. In the thin-film limit $(t \ll D)$, the dispersion relations reduce to $\mu_{y y}{ }^{\text {eff }}(\omega)=0$, so the modes become nondispersive. In the parallel configuration, $\mu_{y y}$ eff $(\omega)=0$ is a biquadratic equation with solutions

$$
\omega_{ \pm}^{2}=\frac{1}{2}\left[\omega_{s}^{2} \pm \sqrt{\omega_{s}^{4}-4\left(\omega_{1}^{2} \omega_{2}^{2}+r(1-r) \Delta \omega^{4}\right.}\right],
$$

where $\omega_{s}^{2}=\omega_{1}^{2}+\omega_{2}^{2}+f_{1} f_{2}(4 \pi \gamma \Delta M)^{2}, \quad \omega_{1,2}^{2}=$ $\gamma^{2} H\left(H+4 \pi M_{1,2}\right), \quad \Delta \omega^{4}=\left(\omega_{2}^{2}-\omega_{1}^{2}\right)^{2}, \quad$ and $\Delta M=$ $M_{2}-M_{1}$. In the transverse configuration, $\mu_{y y}{ }^{\text {eff }}(\omega)=0$ reduces to the quadratic form with a positive root, which is the root-mean-square of $\omega_{1}$ and $\omega_{2}$ :

$$
\omega_{0}=\sqrt{r \omega_{1}^{2}+(1-r) \omega_{2}^{2}}
$$

In the two-frequency regime, we see that the superlattice frequencies $\omega_{+}$and $\omega_{-}$differ from $\omega_{1}$ and $\omega_{2}$, which would be in the individual layers with magnetizations $M_{1}$ and $M_{2}$, i.e., $\omega_{+}>\omega_{2}>\omega_{0}>\omega_{1}>\omega_{-}$[Fig. 4(a)]. Thus, interstripe interactions cause broadening of the bandpass from $\omega_{2}-\omega_{1}$ to $\omega_{+}-\omega_{-}$.

Figure 4(b) shows the calculated frequencies as functions of $H$ with $M_{1}=0.25 \mathrm{kG}$ and $M_{2}=0.45 \mathrm{kG}$ (lower band) as well as $M_{1}=1.1 \mathrm{kG}$ and $M_{2}=1.3 \mathrm{kG}$ (upper band). These values are compared with measured frequencies (shown by symbols) in patterned Fe-V samples at the two edges of the range of $\mathrm{Fe}$ concentration $(65 \%$ and $70 \%)$. We find that, in the two-frequency regime, the measured frequencies of the upper and lower peaks coincide, respectively, with $\omega_{+}$and $\omega_{-}$(see also the inset of Fig. 3).

Finally, we address the question of why our systems do exhibit such unusual properties associated with achieving the LWL. Because of spin pinning at the interfaces, it is hard to expect that the proposed mechanism of interstripe coupling would be effective. For a better understanding of this paradox, in particular, it can be useful to take into account intrastripe exchange, which comes into play in the submicron structures by making a more uniform distribution of the dynamic fields in the lowest precession mode and the spins unpinned at the interfaces. In fact, if we increase the lateral sizes of our structures up to a few micron or above, we lose the collective effects described here. However, in our studies of larger structures we cannot provide sizes of the pump spot that would be much larger than $D$ under the current experimental conditions.

In summary, we have observed pronounced collective effects on spin dynamics in lateral magnetic superlattices. The observed features are explainable in terms of the longwavelength approximation by involving the nonevanescent dipolar fields generated by the nonuniform spin precession. This mechanism of interstripe coupling can be effective in the absence of interfacial spin pinning and interstripe exchange coupling. The observed behaviors can exist in other (nonmagnetic) types of superlattices. Another point is that the collective effects, which we have found in our structures, make them promising to be applied in novel microwave devices with a $\boldsymbol{H}$-orientation-driven bandpass in a frequency range above $10 \mathrm{GHz}$, which is of great interest for different applications, both civilian and military.

This work was supported by NSF MRSEC, the W. M. Keck Foundation, and RFBR (No. 04-02-17048).

[1] L. Esaki, in Novel Materials and Techniques in Condensed Matter, edited by G. W. Crabtree and P. Vashishta (NorthHolland, New York, 1982), p. 1.

[2] G. F. Giuliani and J. J. Quinn, Phys. Rev. Lett. 51, 919 (1983); H. A. Fertig and S. Das Sarma, Phys. Rev. Lett. 65, 1482 (1990); A. K. Sood et al., Phys. Rev. Lett. 54, 2115 (1985); J.R. Dutcher et al., Phys. Rev. Lett. 68, 2464 (1992).

[3] M. G. Cottam and D. R. Tilley, Introduction to Surface and Superlattice Excitations (Cambridge University Press, Cambridge, 1989).

[4] D. L. Mills, in Light Scattering in Solids V, edited by M. Cardona and G. Güntherodt (Springer, Berlin, 1989), Chap. 2.

[5] R. E. Camley, T. S. Rahman, and D. L. Mills, Phys. Rev. B 27, 261 (1983).

[6] P. Grünberg and K. Mika, Phys. Rev. B 27, 2955 (1983).

[7] M. Grimsditch et al., Phys. Rev. Lett. 51, 498 (1983).

[8] R. Arias and D. L. Mills, Phys. Rev. B 67, 094423 (2003); 60, 7395 (1999).

[9] N. Raj and D. R. Tilley, Phys. Rev. B 36, 7003 (1987).

[10] X.-Z. Wang and D. R. Tilley, Phys. Rev. B 50, 13472 (1994).

[11] C. Kittel, Phys. Rev. 110, 1295 (1958); M. Sparks, Phys. Rev. B 1, 3831 (1970).

[12] K. Yu. Guslienko et al., Phys. Rev. B 66, 132402 (2002).

[13] V.M. Agranovich and V.E. Kravtsov, Solid State Commun. 55, 85 (1985).

[14] N. Raj and D. R. Tilley, Solid State Commun. 55, 373 (1985); P. Halevi, A. A. Krokhin, and J. Arriaga, Phys. Rev. Lett. 82, 719 (1999).

[15] N. I. Polushkin et al., J. Appl. Phys. 81, 5478 (1997); M. Zheng et al., Appl. Phys. Lett. 79, 2606 (2001); N. I. Polushkin, Appl. Phys. Lett. 86, 132502 (2005).

[16] B. K. Kuanr et al., Appl. Phys. Lett. 87, 222506 (2005), and references therein.

[17] Y. Blyakhman et al., Phys. Rev. B 52, 10303 (1995).

[18] M. van Campen et al., Phys. Rev. Lett. 88, 227201 (2002).

[19] R. W. Damon and J. R. Eshbach, J. Phys. Chem. Solids 19, 308 (1961). 